\title{
Numerical Analysis of Vibration Isolation Using Pile Rows against the Vibration due to Moving Loads in a Viscoelastic Medium
}

\author{
Bin Xu and Man-Qing Xu \\ Department of Civil Engineering, Nanchang Institute of Technology, Nanchang, Jiangxi 330029, China \\ Correspondence should be addressed to Bin Xu; xmq418@163.com
}

Received 1 April 2014; Revised 20 July 2014; Accepted 24 July 2014; Published 8 September 2014

Academic Editor: Viktor Popov

Copyright (C) 2014 B. Xu and M.-Q. Xu. This is an open access article distributed under the Creative Commons Attribution License, which permits unrestricted use, distribution, and reproduction in any medium, provided the original work is properly cited.

\begin{abstract}
A numerical method for evaluating the vertical vibration isolation effect of pile rows embedded in a viscoelastic half space subjected to a moving load is developed in this paper on the basis of the Cole-Cole model and Muki's method. Based on the proposed method, the influence of various parameters on the vibration isolation effect of pile rows embedded in the viscoelastic half space is investigated numerically.
\end{abstract}

\section{Introduction}

Vibration induced by railway traffic is a major concern for civil engineers as it causes annoyance to residents or even damage to adjacent structures. Generally, the effects of ground vibrations can be mitigated by two kinds of vibration isolation methods: the active and the passive vibration isolation methods. The active isolation system is often used to reduce the ground vibration near the source. It is usually installed either around the vibration source or at a close distance to the source. The passive isolation system, on the other hand, usually is far away from the source and surrounds the protected structure. Normally, there are two passive vibration isolation methods: the trench (open or infilled) isolation method and the pile (pile rows or sheet piles) isolation method. To date, many studies concerning vibration isolation using trenches or piles have been conducted. For example, Emad and Manolis [1] utilized the boundary element method (BEM) with constant elements to examine the efficiency of vibration reduction by open trench with a rectangular or a circular cross-section. Considering the coupling effects between the soil skeleton and underground water, Cao et al. [2] proposed an analytical model to investigate the screening efficiency of trenches to moving-load induced ground vibrations based on Biot's dynamic poroelastic theory. Cai et al. [3] investigated the vibration isolation effect of pile rows embedded in a poroelastic medium by using the wave function expansion method. Kattis et al. [4,5] used 3D BEM to calculate the screening effectiveness of a pile row in the frequency domain. Also, by means of the frequency domain BEM, the screening effectiveness of four types of circular piles in a row against the vibration due to a massless square foundation subjected to a harmonic vertical loading is studied by Tsai et al. [6]. Besides, by means of the fictitious pile method developed by Muki and Sternberg [7] and the direct superposition method, Lu et al. [8,9] analyzed the vibration isolation effect of pile rows.

It is noticed that previous studies concern the vibration isolation modelling in a generalized standard linear viscoelastic solid. For the generalized standard linear viscoelastic solid, the complex modulus belongs to the rational function. However, both creep tests [10] and vibration tests [11] suggest that the derivatives of stress relaxation functions have an asymptotic behaviour const. As a result, the convolution in the constitutive relation of the heterogeneous viscoelastic medium cannot be eliminated by the method used in the generalized standard linear viscoelastic solid. Unlike the standard linear solid, the complex modulus of the Cole-Cole viscoelastic medium is not the rational function, a viscoelastic kernel having a singularity const $\times t^{-\alpha}$ at $t \rightarrow 0$, where $0<\alpha<1$, is used to characterize the viscoelastic medium [12]. The Cole-Cole model has been validated experimentally 
in both quasi-static and vibrations experiments [10]. It has been pointed out in several studies, for example Bagley and Torvik [13], Lu and Hanyga [14], and Soula et al. [15], that the Cole-Cole model fits experimental data over several decades of frequency. Experimental evidences in rock physics [16, 17] also point out to the Cole-Cole type behavior. Furthermore, the Cole-Cole relaxation model has many advantages over other models. In particular, the Cole-Cole relaxation model is compatible with a finite speed of wave propagation. It is very important to conduct accurate analysis of the wave attenuation and dispersion when moving loads are of high velocities.

In this study, a numerical method for evaluating the vertical vibration isolation effect of pile rows embedded in a viscoelastic half space subjected to a moving load is developed on the basis of the Cole-Cole model [12] and Muki's method [7]. Based on the proposed method, the influence of various parameters on the vibration isolation effect of pile rows embedded in the viscoelastic half space is investigated numerically. It is noted that the proposed method in this study belongs to the semianalytical category. Thus, compared with conventional domain discretization methods such as the finite element method (FEM) and the boundary element method (BEM), it significantly reduces the computational time.

\section{The Free Wave Field Solution and the Fundamental Solution for a Circular Uniform Patch Load}

Usually, for a linear isotropic viscoelastic medium described by the Cole-Cole model, the $P$ and the $S$ wave modes satisfy the Cole-Cole relaxation law with different parameters. Consequently, in terms of the Cole-Cole models, the complex moduli for the $P$ and the $S$ waves in the frequency domain have the following forms:

$$
\begin{aligned}
M_{p}(\omega) & =\bar{\lambda}(\omega)+2 \bar{\mu}(\omega) \\
& =M_{\infty p} \frac{1+a_{p}\left(i \eta_{p} \omega\right)^{-\alpha_{p}}}{1+\left(i \eta_{p} \omega\right)^{-\alpha_{p}}}=M_{\infty p}+\frac{M_{\infty p}\left(a_{p}-1\right)}{1+\left(i \eta_{p} \omega\right)^{-\alpha_{p}}}
\end{aligned}
$$

$$
\begin{aligned}
M_{s}(\omega) & =\bar{\mu}(\omega) \\
& =M_{\infty} \frac{1+a_{s}\left(i \eta_{s} \omega\right)^{-\alpha_{s}}}{1+\left(i \eta_{s} \omega\right)^{-\alpha_{s}}}=M_{\infty s}+\frac{M_{\infty s}\left(a_{s}-1\right)}{1+\left(i \eta_{s} \omega\right)^{-\alpha_{s}}},
\end{aligned}
$$

where a bar over the function denotes the Fourier transform for time $t \rightarrow$ frequency $\omega$. The subscripts $p$ and $s$ denote the $P$ and $S$ waves in a viscoelastic medium and $\bar{\lambda}(\omega)$ and $\bar{\mu}(\omega)$ are the complex modulus corresponding to the two Lame constants. $M_{\infty}$ is the limit of the complex modulus for $\omega \rightarrow$ $\infty, M_{0}$ is the value of the complex modulus for $\omega=0, \eta$ is a characteristic relaxation time, and $\alpha$ controls the width of the transition zone between $M_{\infty}$ and $M_{0}$. Besides, the conditions $0<\alpha<1$ and $a \leq 1$ follow from thermodynamics argument [12].

In this study, the free wave field solution is defined as the solution of the moving load in the absence of the pile rows. For a moving load, axisymmetry is lost due to the orientation of the load speed; thus, it is more convenient to consider the moving load problem in the Cartesian coordinate system (Figure 1). A moving load with a constant speed $c$ and an oscillating frequency $\omega_{0}$ is applied on the surface of the viscoelastic half space. The load moves along the negative direction of the $y$-axis and the distance between the load and $y$-axis is $d_{s}$ (Figure 1). For the moving load applied over a rectangular area $2 a \times 2 b$, the boundary conditions in the time-space domain are as follows:

$$
\begin{gathered}
\left.\sigma_{z x}(x, y, z, t)\right|_{z=0}=0 \\
\left.\sigma_{z y}(x, y, z, t)\right|_{z=0}=0 \\
\left.\sigma_{z z}(x, y, z, t)\right|_{z=0} \\
=-q_{z}\left[H\left(x+d_{s}+a\right)-H\left(x+d_{s}-a\right)\right] \\
\times\left[H\left(y-y_{0}+b+c t\right)-H\left(y-y_{0}-b+c t\right)\right] e^{i \omega_{0} t},
\end{gathered}
$$

where $q_{z}$ is the intensity of the distributed load, $\omega_{0}$ is the frequency of moving load, $H(*)$ is the Heaviside step function, and $y_{0}$ is the $y$ coordinate of the center of the distributed load at $t=0$.

Performing a triple Fourier transform with respect to time and the two horizontal coordinates on $((2 \mathrm{a}),(2 \mathrm{~b})$, and (2c)), respectively, the following boundary conditions are derived in the frequency wavenumber domain:

$$
\begin{gathered}
\widetilde{\overline{\bar{\sigma}}}_{z x}\left(k_{x}, k_{y}, 0, \omega\right)=0 \\
\widetilde{\bar{\sigma}}_{z y}\left(k_{x}, k_{y}, 0, \omega\right)=0 \\
\widetilde{\overline{\bar{\sigma}}}_{z z}\left(k_{x}, k_{y}, 0, \omega\right) \\
=-8 \pi q_{z} \frac{\sin \left(k_{x} a\right)}{k_{x}} \frac{\sin \left(k_{y} b\right)}{k_{y}} e^{i\left(k_{x} d_{s}-k_{y} y_{0}\right)} \delta\left(\omega-\omega_{0}-k_{y} c\right)
\end{gathered}
$$

in which $k_{x}$ and $k_{y}$ represent the two horizontal wave numbers corresponding to $x$ - and $y$-coordinates, respectively, and $\delta(*)$ is the Dirac delta function.

For a moving point load, the boundary conditions for $\sigma_{z x}, \sigma_{z y}$ are the same as those for the moving rectangular distributed load, while the boundary condition for $\sigma_{z z}$ is as follows

$$
\left.\sigma_{z z}(x, y, z, t)\right|_{z=0}=-F_{z} \delta\left(x+d_{s}\right) \delta\left(y-y_{0}+c t\right) e^{i \omega_{0} t} .
$$

Likewise, the boundary condition for $\widetilde{\bar{\sigma}}_{z z}$ in the frequency wavenumber domain is given by

$$
\widetilde{\bar{\sigma}}_{z z}\left(k_{x}, k_{y}, 0, \omega\right)=-2 \pi F_{z} e^{i\left(k_{x} d_{s}-k_{y} y_{0}\right)} \delta\left(\omega-\omega_{0}-c k_{y}\right) .
$$




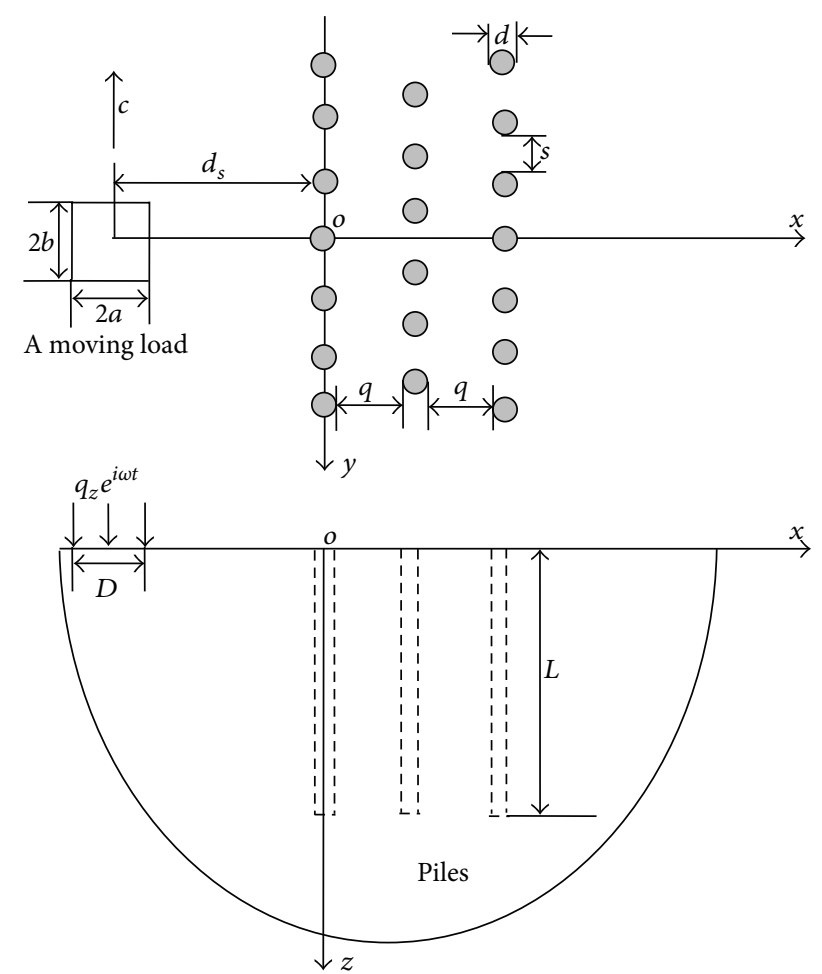

FIGURE 1: Piles rows embedded in a viscoelastic half space as the vibration isolation system against the vibration due to moving loads.

Using ((3a), (3b), and (3c)) or (5) as well as the expressions for the stress in the frequency wavenumber domain, the free wave field due to the surface moving load can be determined. In view of (3a), (3b), and (3c), all the variables in the frequency wavenumber domain due to the moving distributed rectangular load can be expressed in the following form:

$$
\begin{aligned}
& \widetilde{\bar{\Omega}}\left(k_{x}, k_{y}, z, \omega\right) \\
& =\left[-8 \pi q_{z} \frac{\sin \left(k_{x} a\right)}{k_{x}} \frac{\sin \left(k_{y} b\right)}{k_{y}} e^{i\left(k_{x} d_{s}-k_{y} y_{0}\right)} \delta\left(\omega-\omega_{0}-k_{y} c\right)\right] \\
& \quad \times \widetilde{\bar{\Omega}}^{*}\left(k_{x}, k_{y}, z, \omega\right),
\end{aligned}
$$

where $\widetilde{\bar{\Omega}}^{*}\left(k_{x}, k_{y}, z, \omega\right)$ is the solution of a variable corresponding to a unit boundary value $\widetilde{\bar{\sigma}}_{z z}$ in (3c). The moving point load has similar expression

$$
\begin{aligned}
& \widetilde{\bar{\Omega}}\left(k_{x}, k_{y}, z, \omega\right) \\
& =\left[-2 \pi F_{z} e^{i\left(k_{x} d_{s}-k_{y} y_{0}\right)} \delta\left(\omega-\omega_{0}-c k_{y}\right)\right] \times \widetilde{\bar{\Omega}}^{*}\left(k_{x}, k_{y}, z, \omega\right) .
\end{aligned}
$$

Performing the inverse Fourier transform with respect to the two horizontal wavenumbers and using the property of

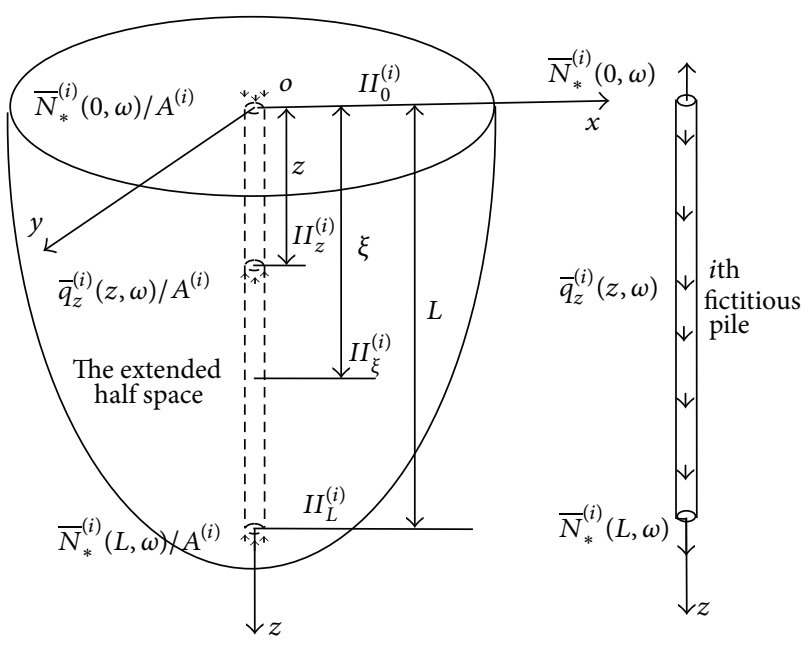

Figure 2: The model for decomposition of the pile-half space system. (a) an extended poro-elastic half-space (b) a fictitious pile.

the delta function, the frequency domain free field solution for the moving distributed rectangular load has the form:

$$
\begin{aligned}
\bar{\Omega}(x, y, z, \omega) \\
=\frac{2 q_{z}}{\pi} \frac{\sin \left[b\left(\omega-\omega_{0}\right) / c\right]}{\omega-\omega_{0}} e^{i\left(\left(\omega-\omega_{0}\right) / c\right)\left(y-y_{0}\right)} \\
\quad \times \int_{-\infty}^{+\infty} \frac{\sin \left(k_{x} a\right)}{k_{x}} \widehat{\bar{\Omega}}^{*}\left(k_{x}, \frac{\omega-\omega_{0}}{c}, z, \omega\right) e^{i k_{x}\left(x+d_{s}\right)} d k_{x} .
\end{aligned}
$$

For the moving point load, the frequency domain free field solution for all variables is represented by

$$
\begin{aligned}
\bar{\Omega}(x, y, z, \omega) \\
=-\frac{F_{z}}{2 \pi c} e^{i\left(\left(\omega-\omega_{0}\right) / c\right)\left(y-y_{0}\right)} \\
\quad \times \int_{-\infty}^{+\infty} \widehat{\bar{\Omega}}^{*}\left(k_{x}, \frac{\omega-\omega_{0}}{c}, z, \omega\right) e^{i k_{x}\left(x+d_{s}\right)} d k_{x} .
\end{aligned}
$$

To establish the integral equations for the pile rows, the frequency domain fundamental solution for a uniform vertical patch load applied in the viscoelastic half space is required. As the problem for a viscoelastic half space subjected to a uniform vertical patch load over a circular area with a radius $R$ (Figure 2) is axisymmetric with respect to the center of the circular area, thus, it is more convenient to consider the problem in the cylindrical coordinate system $(r, \theta, z)$.

For a uniform vertical patch load applied in the viscoelastic half space, the surface of the half space is stress-free. Therefore, the boundary conditions for the half-space surface are as follows:

$$
\widetilde{\widetilde{\sigma}}_{z z}^{[0]}(\xi, 0, \omega)=0, \quad \widetilde{\bar{\sigma}}_{z r}^{[1]}(\xi, 0, \omega)=0,
$$

where $[m]$ denotes the $m$ th order first kind of Bessel function and $\xi$ denotes the Hankel transform parameter. 
It is assumed that the circular patch load is applied at the depth $z=h$. Accordingly, the half space is divided by the plane $z=h$ into an upper and a lower region and, therefore, the continuity conditions at the plane $z=h$ are as follows:

$$
\begin{gathered}
\widetilde{\bar{u}}_{r}^{[1]}\left(\xi, h^{-}, \omega\right)=\widetilde{\bar{u}}_{r}^{[1]}\left(\xi, h^{+}, \omega\right), \\
\widetilde{\bar{u}}_{z}^{[0]}\left(\xi, h^{-}, \omega\right)=\widetilde{\bar{u}}_{z}^{[0]}\left(\xi, h^{+}, \omega\right), \\
\widetilde{\bar{\sigma}}_{z z}^{[0]}\left(\xi, h^{+}, \omega\right)-\widetilde{\bar{\sigma}}_{z z}^{[0]}\left(\xi, h^{-}, \omega\right)=-\frac{R J_{1}(R \xi)}{A \xi}, \\
\widetilde{\bar{\sigma}}_{z r}^{[1]}\left(\xi, h^{-}, \omega\right)=\widetilde{\bar{\sigma}}_{z r}^{[1]}\left(\xi, h^{+}, \omega\right) .
\end{gathered}
$$

Using the general expressions for the displacement and the stress and (10) and (11), the arbitrary constants involved in the expressions for the potentials, the displacement, and the stress can be determined. The frequency fundamental solution can be obtained by performing inverse Hankel transform on the solutions for the displacement, the stress in the frequency-wavenumber domain [18].

\section{Fredholm Integral Equations Describing Dynamic Interaction between Piles and the Viscoelastic Half Space}

As shown in Figure 1, the pile rows embedded in the viscoelastic half space are used to isolate the vibration generated by a moving load. The number of the total pile is $m=\sum_{k=1}^{K} n_{k}$, where $K$ and $n_{k}$ denote the number of pile rows and the number of the piles in the $k$ th row. The spacing between two neighboring piles in each pile row is denoted by $s$. The spacing between two neighboring pile rows is $q$. Also, it is assumed that each pile has the same diameter $d(d=2 R)$ and the same length $L(d / L \ll 1)$. A moving load with a constant speed $c$ and an oscillating frequency $\omega_{0}$ is acting on the surface of the viscoelastic half space and moves along the negative direction of the $y$-axis (Figure 1).

When the pile-half-space system is subjected to a vertical moving load, generally, the pile will undergo both vertical and horizontal response. However, as the influence of the horizontal interaction between the pile-soil system is relatively smaller, the horizontal interaction between piles and the half space is neglected in this paper. Thus, only the vertical interaction between the piles and the half space is considered in the paper.

Following Muki and Sternberg [7] and Pak and Jennings [19], the current problem is decomposed into two subproblems: an extended viscoelastic half space and the multiple fictitious piles. The extended viscoelastic half space is described by the continuum dynamic theory for a viscoelastic medium, while the fictitious piles are described by the 1D bar vibration theory. The decomposition procedure is illustrated in Figure 2 by the $i$ th pile of the pile rows.

It is assumed that the axial force of the $i$ th fictitious pile is $\bar{N}_{*}^{(i)}(z, \omega)$ and the vertical distributed load along the $i$ th fictitious pile is $\bar{q}_{z}^{(i)}(z, \omega)$ (Figure 2(b)). The top and the bottom of the ith fictitious pile are subjected to forces $\bar{N}_{*}^{(i)}(0, \omega)$ and $\bar{N}_{*}^{(i)}(L, \omega)$, respectively. The viscoelastic half space is subjected to the following loads (Figure 2(a)): $\bar{q}_{z}^{(i)}(z, \omega)$ which is distributed over the region occupied by the $i$ th pile; $\bar{N}_{*}^{(i)}(0, \omega) / A^{(i)}$ and $\bar{N}_{*}^{(i)}(L, \omega) / A^{(i)}$ which are applied to the circular areas $\Pi_{0}^{(i)}$ and $\Pi_{L}^{(i)}$, respectively. Note that $A^{(i)}$ denotes the cross-section area of the $i$ th pile.

For the ith fictitious pile, the displacement $\bar{u}_{z p^{*}}^{(i)}(z, \omega)$, the distributed vertical force $\bar{q}_{z}^{(i)}(z, \omega)$, and the axial force satisfy the following relations:

$$
\begin{array}{r}
\bar{q}_{z}^{(i)}(z, \omega)=-\frac{d \bar{N}_{*}^{(i)}(z, \omega)}{d z}-\rho_{p^{*}}^{(i)} A^{(i)} \omega^{2} \bar{u}_{z p^{*}}^{(i)}(z, \omega), \\
i=1,2, \ldots, m, \\
\bar{u}_{z p^{*}}^{(i)}(z, \omega)=\bar{u}_{z p^{*}}^{(i)}(0, \omega)+\frac{1}{E_{p^{*}}^{(i)} A^{(i)}} \int_{0}^{z} \bar{N}_{*}^{(i)}(\eta, \omega) d \eta, \\
i=1,2, \ldots, m
\end{array}
$$

in which $\bar{u}_{z p^{*}}^{(i)}(z, \omega)$ is the vertical displacement of the $i$ th pile.

The vertical strain of the extended half space along the axis of the ith pile is composed of two parts: the first part is due to the free wave field, while the second part is due to the force applied to the extended half space by the fictitious piles. Thus, the vertical strain of the extended half space along the axis of the $i$ th pile can be written as

$$
\begin{aligned}
& \bar{\varepsilon}_{z s}^{(i)}(z, \omega)= \bar{\varepsilon}_{z f}^{(i)}(z, \omega) \\
&+\sum_{j=1}^{m}\left[\bar{N}_{*}^{(j)}(0, \omega) \bar{\varepsilon}_{z}^{(G)}\left(r_{i j}, 0, z, \omega\right)\right. \\
& \quad-\bar{N}_{*}^{(j)}(L, \omega) \bar{\varepsilon}_{z}^{(G)}\left(r_{i j}, L, z, \omega\right) \\
&\left.\quad-\int_{0}^{L_{j}} \bar{q}_{z}^{(j)}(\zeta, \omega) \bar{\varepsilon}_{z}^{(G)}\left(r_{i j}, \zeta, z, \omega\right) d \zeta\right], \\
& \quad i=1,2, \ldots, m .
\end{aligned}
$$

In (13), the superscript and subscript $i$ and $j$ denote the $i$ th and the $j$ th pile, respectively, $\bar{\varepsilon}_{z f}^{(i)}(z, \omega)$ is the free field vertical strain at the axis of the $i$ th pile, which is determined by the free field frequency domain solution for the moving load, and $\bar{\varepsilon}_{z}^{(G)}\left(r_{i j}, \zeta, z, \omega\right)$ represents the vertical strain at the center of $\Pi_{z}^{(i)}$ due to a unit patch load applied at $\Pi_{\zeta}^{(j)}$ (Figure $2(\mathrm{a})$ ) and $r_{i j}$ is the horizontal distance between the axis of the $i$ th and $j$ th pile. It is worth noting that, for the case $i=j, r_{i j}$ is vanishing.

Using (12a) and (12b) and (13), the following relation is obtained

$$
\begin{aligned}
& \bar{\varepsilon}_{z s}^{(i)}(z, \omega) \\
& =\bar{\varepsilon}_{z f}^{(i)}(z, \omega) \\
& \quad-\bar{N}_{*}^{(i)}(z, \omega)\left[\bar{\varepsilon}_{z}^{(G)}\left(r_{i i}, z^{+}, z, \omega\right)-\bar{\varepsilon}_{z}^{(G)}\left(r_{i i}, z^{-}, z, \omega\right)\right]
\end{aligned}
$$




$$
\begin{aligned}
& -\int_{0}^{L_{i}} \bar{N}_{*}^{(i)}(z, \omega) \frac{\partial \bar{\varepsilon}_{z}^{(G)}\left(r_{i i}, \zeta, z, \omega\right)}{\partial \zeta} d \zeta \\
& +\rho_{p^{*}}^{(i)} A^{(i)} \omega^{2} \int_{0}^{L_{i}} \bar{u}_{z p^{*}}^{(i)}(\zeta, \omega) \bar{\varepsilon}_{z}^{(G)}\left(r_{i i}, \zeta, z, \omega\right) d \zeta \\
& +\sum_{j=1}^{m(j \neq i)}\left[-\int_{0}^{L_{j}} \bar{N}_{*}^{(j)}(\zeta, \omega) \frac{\partial \bar{\varepsilon}_{z}^{(G)}\left(r_{i j}, \zeta, z, \omega\right)}{\partial \zeta} d \zeta\right. \\
& \left.+\rho_{p^{*}}^{(j)} A^{(j)} \omega^{2} \int_{0}^{L_{j}} \bar{u}_{z p^{*}}^{(j)}(\zeta, \omega) \bar{\varepsilon}_{z}^{(G)}\left(r_{i j}, \zeta, z, \omega\right) d \zeta\right]
\end{aligned}
$$

where $\bar{\varepsilon}_{z}^{(G)}\left(r_{i i}, z^{-}, z, \omega\right)$ and $\bar{\varepsilon}_{z}^{(G)}\left(r_{i i}, z^{+}, z, \omega\right)$ denote the vertical strain of the viscoelastic half space at the center of $\Pi_{z}^{(i)}$ of the $i$ th pile when the patch load $\Pi_{\xi}^{(i)}$ approaches $\Pi_{z}^{(i)}$ from up and down side, respectively.

In this study, the compatibility condition between the $i$ th pile and the viscoelastic half space is fulfilled by requiring the vertical strain of the $i$ th fictitious pile and that of the extended half space along the axis of the $i$ th fictitious pile to be equal to

$$
\bar{\varepsilon}_{z p^{*}}^{(i)}(z, \omega)=\bar{\varepsilon}_{z s}^{(i)}(z, \omega), \quad 0 \leq z \leq L, i=1,2, \ldots, m,
$$

where $\bar{\varepsilon}_{z p^{*}}^{(i)}(z, \omega)$ represents the vertical strain of the $i$ th fictitious pile.

Using (12a) and (12b), (14) and (15), the Fredholm integral equation in the frequency domain describing the vertical interaction between the $i$ th pile and the half space has the form

$$
\begin{aligned}
& \frac{\bar{N}_{*}^{(i)}(z, \omega)}{E_{p^{*}}^{(i)} A^{(i)}} \\
& +\bar{N}_{*}^{(i)}(z, \omega)\left[\bar{\varepsilon}_{z}^{(G)}\left(r_{i i}, z^{+}, z, \omega\right)-\bar{\varepsilon}_{z}^{(G)}\left(r_{i i}, z^{-}, z, \omega\right)\right] \\
& +\sum_{j=1}^{m}\left[\int_{0}^{L_{j}} \bar{N}_{*}^{(j)}(\zeta, \omega) \frac{\partial \bar{\varepsilon}_{z}^{(G)}\left(r_{i j}, \zeta, z, \omega\right)}{\partial \zeta} d \zeta\right. \\
& \quad-\int_{0}^{L_{j}} \bar{N}_{*}^{(j)}(\zeta, \omega) \bar{\chi}_{i j}^{(a)}(\zeta, z, \omega) d \zeta \\
& \left.\quad-\bar{u}_{z p^{*}}^{(j)}(0, \omega) \bar{\chi}_{i j}^{(b)}(z, \omega)\right] \\
& =\bar{\varepsilon}_{z f}^{(i)}(z, \omega), \quad i=1,2, \ldots, m,
\end{aligned}
$$

where

$$
\begin{gathered}
\bar{\chi}_{i j}^{(a)}(\zeta, z, \omega)=\left(\frac{\rho_{p^{*}}^{(j)} \omega^{2}}{E_{p^{*}}^{(j)}}\right) \int_{\zeta}^{L_{j}} \bar{\varepsilon}_{z}^{(G)}\left(r_{i j}, \eta, z, \omega\right) d \eta, \\
\bar{\chi}_{i j}^{(b)}(z, \omega)=\rho_{p^{*}}^{(j)} A^{(j)} \omega^{2} \int_{0}^{L_{j}} \bar{\varepsilon}_{z}^{(G)}\left(r_{i j}, \eta, z, \omega\right) d \eta .
\end{gathered}
$$

Following the similar procedures, the surface vertical displacement $\bar{u}_{z}\left(\mathbf{x}_{\perp}, z=0, \omega\right)$ for the viscoelastic half space in the presence of the pile rows can be calculated as follows:

$$
\begin{aligned}
& \bar{u}_{z}\left(\mathbf{x}_{\perp}, 0, \omega\right) \\
& =\bar{u}_{z f}\left(\mathbf{x}_{\perp}, 0, \omega\right) \\
& +\sum_{j=1}^{m}\left[-\int_{0}^{L_{j}} \bar{N}_{*}^{(j)}(\zeta, \omega) \frac{\partial \bar{u}^{(G)}\left(r_{\mathbf{x}_{\perp} j}, \zeta, 0, \omega\right)}{\partial \zeta} d \zeta\right. \\
& \left.\quad+\rho_{p^{*}}^{(j)} A^{(j)} \omega^{2} \int_{0}^{L_{j}} \bar{u}_{z p^{*}}^{(j)}(\zeta, \omega) \bar{u}^{(G)}\left(r_{\mathbf{x}_{\perp} j}, \zeta, 0, \omega\right) d \zeta\right],
\end{aligned}
$$

where $\bar{u}_{z f}^{(S)}\left(\mathbf{x}_{\perp}, 0, \omega\right)$ represents the free field vertical displacement, $\bar{u}^{(G)}\left(r_{\mathbf{x}_{\perp} j}, \zeta, 0, \omega\right)$ denotes the vertical displacement at the surface point $\mathbf{x}_{\perp}\left(\mathbf{x}_{\perp}=x i+y j\right)$ due to a unit patch load applied at $\Pi_{\xi}^{(j)}$, and $r_{\mathbf{x}_{\perp} j}$ is the horizontal distance between the surface point $\mathbf{x}_{\perp}$ and the axis of the $j$ th pile.

In (11), the vertical displacement of the $i$ th pile top $\bar{u}_{z p^{*}}^{(i)}(0, \omega)$ is also unknown. The unknown $\bar{u}_{z p^{*}}^{(i)}(0, \omega)$ can be represented by the axial force of the fictitious piles if the vertical displacement of the ith pile top and the surface vertical displacement of the extended half space at the $i$ th pile top are assumed to be equal; that is, $\bar{u}_{z p^{*}}^{(i)}(0, \omega)=\bar{u}_{z}^{(i)}(0, \omega)$. Note that $\bar{u}_{z}^{(i)}(0, \omega)$ can be obtained via (18) by setting $\mathbf{x}_{\perp}$ coinciding with the center of the ith pile. Thus, using (12a) and (12b) and (18), the following supplementary equations for $\bar{u}_{z p^{*}}^{(i)}(0, \omega)$ are derived:

$$
\begin{aligned}
\sum_{j=1}^{m}[- & \left.\int_{0}^{L_{j}} \bar{N}_{*}^{(j)}(\zeta, \omega) \frac{\partial \bar{u}^{(G)}\left(r_{i j}, \zeta, 0, \omega\right)}{\partial \zeta}\right] d \zeta \\
& +\sum_{j=1}^{m} \int_{0}^{L_{j}} \bar{N}_{*}^{(j)}(\zeta, \omega) \bar{\chi}_{i j}^{(c)}(\zeta, 0, \omega) d \zeta \\
& +\sum_{j=1}^{m} \bar{u}_{z p^{*}}^{(j)}(0, \omega)\left[\bar{\chi}_{i j}^{(d)}(0, \omega)-\delta_{i j}\right] \\
= & -\bar{u}_{z f}^{(i)}(0, \omega), \quad i=1,2, \ldots, m,
\end{aligned}
$$

where $\delta_{i j}$ is the Kronecker delta and

$$
\begin{aligned}
& \bar{\chi}_{i j}^{(c)}(\zeta, z, \omega)=\frac{\rho_{p j^{*}}^{(j)} \omega^{2}}{E_{p j^{*}}^{(j)}} \int_{\zeta}^{L_{j}} \bar{u}^{(G)}\left(r_{i j}, \eta, z, \omega\right) d \eta, \\
& \bar{\chi}_{i j}^{(d)}(z, \omega)=\rho_{p^{*}}^{(j)} A^{(j)} \omega^{2} \int_{0}^{L_{j}} \bar{u}^{(G)}\left(r_{i j}, \eta, z, \omega\right) d \eta .
\end{aligned}
$$

\section{Numerical Results and Discussions}

The integral equations in the frequency domain accounting for the vertical interaction between pile rows and the half 
space can be solved numerically. The methodology for solving integral equation (15) was detailed in [19]. After discretization of (16) and (19), the following linear algebraic equations in the frequency domain are obtained:

$$
\mathbf{A}(\omega) \mathbf{X}(\omega)=\mathbf{b}(\omega)
$$

where $\mathbf{A}(\omega)$ is the coefficient matrix determined by discrete integral equations which is associated with the fundamental solution, $\mathbf{b}(\omega)$ is the right-handed term which is determined by the free field solution, such as $\bar{\varepsilon}_{z}^{(f)}(x, y, z, \omega)$, and $\mathbf{X}(\omega)$ is the discrete unknowns of the integral equations.

In order to recover the solution in the time domain, a series of frequency domain solutions at discrete sample points need to be determined first. Assuming the number of the frequency domain sample points is $2 N+1$, then (21) for the sample points $i=1,2, \ldots, N, N+1$ has the following form:

$$
\begin{array}{r}
\left.\left.\mathbf{A}(\omega)\right|_{\omega=(i-1) \Delta \omega} \mathbf{X}(\omega)\right|_{\omega=(i-1) \Delta \omega}=\left.\mathbf{b}(\omega)\right|_{\omega=(i-1) \Delta \omega}, \\
i=1,2, \ldots, N, N+1,
\end{array}
$$

where $\Delta \omega$ is the frequency increment for the sample points in the frequency domain and is given by

$$
\Delta \omega=\frac{2 \pi}{T}, \quad T=\frac{2 y_{0}}{c} .
$$

Due to the vibration frequency $\omega_{0}$ of the moving load in (2a), (2b), and (2c) and (4), the right-handed term $\mathbf{b}(\omega)$ for the sample points $i=N+2, \ldots, 2 N+1$ should be determined by the following equation:

$$
[\mathbf{b}(\omega)]_{i}=\left.\mathbf{b}(\omega)\right|_{\omega=-[(2 N+2)-i] \Delta \omega}, \quad i=N+2, \ldots, 2 N+1 .
$$

After numerical solution of the integral equation (16) and (19) for the sample points $i=1,2, \ldots, N+1, N+2, \ldots, 2 N+1$, all the variables in the frequency domain are obtained. The time domain solution for the variables can be obtained by performing inverse Fourier transform on the corresponding frequency domain solutions, which is implemented by the FFT method in this study [20].

For verification purposes, Section 4.1 demonstrates the comparison between the solution of a special case from the proposed method and published results. In Section 4.2, some numerical examples and corresponding analysis are presented.

4.1. Comparison of Our Results with Known Results. In this section, the method developed in this study is justified by comparing a special case of our model with existing results. As shown in Figure 1, the vibration source is a moving distributed load with a constant speed $c$ in the negative direction of the $y$-axis. The intensity of the load is $100 \mathrm{kN}$, and it is uniformly distributed over a rectangle region $2 a \times 2 b=$ $0.8 \mathrm{~m} \times 0.8 \mathrm{~m}$ with a vibration frequency $f=50 \mathrm{~Hz}$. A single 8 -pile row with circular cross-sections is used as the passive isolation vibration system. All piles are featured by a diameter of $d=1.0 \mathrm{~m}$, a length of $L=5.0 \mathrm{~m}$, Young's modulus of $E_{p}=$
$3.3 \times 10^{10} \mathrm{~N} / \mathrm{m}^{2}$, and a density of $\rho_{p}=2.4 \times 10^{3} \mathrm{~kg} / \mathrm{m}^{3}$. The net spacing between two neighboring piles is $s=0.5 \mathrm{~m}$. The distance between $y$-axis and the moving load is $d_{s}=7.5 \mathrm{~m}$.

According to [14], if the parameters $a_{k}(k=p, s)$ for the viscoelastic half space are assumed to tend to 1 , then the viscoelastic half space is reduced to an elastic half space. Moreover, if the speed of the vertical moving distributed load approaches zero, the moving vibration load is reduced to a fixed time-harmonic force. In this paper, the parameter for the viscoelastic is $a_{p}=a_{s}=0.98, \alpha_{p}=\alpha_{s}=0.5, \eta_{p}=\eta_{s}=$ $0.1 \mathrm{~s}, M_{\infty s}=1.32 \times 10^{8} \mathrm{~N} / \mathrm{m}^{2}, M_{\infty p}=3.96 \times 10^{8} \mathrm{~N} / \mathrm{m}^{2}$, and $\rho=2.0 \times 10^{3} \mathrm{~kg} / \mathrm{m}^{3}$. The moving load speed is $c=0.001 \mathrm{~m} / \mathrm{s}$. The corresponding vibration isolation against the fixed timeharmonic force using pile rows in an elastic medium was reported by Kattis et al. [5]. In calculation, the wavelength for the Rayleigh wave of the reduced elastic medium is $\lambda_{R}=$ $5.0 \mathrm{~m}$.

To assess the vibration isolation effect of pile rows, the amplitude reduction ratio $A_{r}$ at point $\mathbf{x}_{\perp}$, which is the ratio between the amplitude of the surface vertical displacement of the half space in the presence of the pile rows and that of the free field solution, is defined as follows:

$$
A_{r}\left(\mathbf{x}_{\perp}, t\right)=\frac{\left|u_{z}\left(\mathbf{x}_{\perp}, z=0, t\right)\right|}{\left|u_{z f}\left(\mathbf{x}_{\perp}, z=0, t\right)\right|}
$$

where $\left|u_{z}\left(\mathbf{x}_{\perp}, z=0, t\right)\right|$ is the amplitude of the vertical displacement of the half space in the presence of the pile rows and $\left|u_{z f}\left(\mathbf{x}_{\perp}, z=0, t\right)\right|$ is the amplitude of the vertical displacement of the soil given by the free field solution.

Woods [21] proposed an average amplitude reduction ratio $\bar{A}_{r}$ for the evaluation of vibration isolation effect, which is defined as follows:

$$
A_{r v}=\frac{1}{A} \int_{A} A_{r} d A,
$$

where $A$ is the rectangle with its width and length determined by a reference length and the width of a pile row. In this study, $A=L_{r} \times \lambda_{R}$, where $L_{r}$ is the width of the first pile row and $\lambda_{R}$ is the wavelength for the Rayleigh wave of the reduced elastic medium.

Figure 3 shows the contour of the amplitude reduction ratio $A_{r}$ for the single pile row embedded in the elastic half space according to the present method when the moving load with a speed $c=0.001 \mathrm{~m} / \mathrm{s}$ is located at the point $(x, y, z)=(-7.5 \mathrm{~m}, 0 \mathrm{~m}, 0 \mathrm{~m})$. It can be observed that the amplitude reduction ratio $A_{r}$ right behind the pile rows is much smaller than those at other areas. According to the calculation in this study, the average amplitude reduction ratio $A_{r v}$ for the elastic half space is 0.718 , while the result of Kattis et al. [5] for the elastic half space is 0.712 . The difference between the present solution and that of Kattis et al. [5] is only $0.842 \%$.

4.2. Numerical Results for the Vibration Isolation of Pile Rows. In this section, the influences of the moving load speeds $(c)$, the number of the pile rows $(K)$, Young's modulus $\left(E_{p}\right)$ of the pile, the pile length $(L)$, and the net spacing $(s)$ between two 


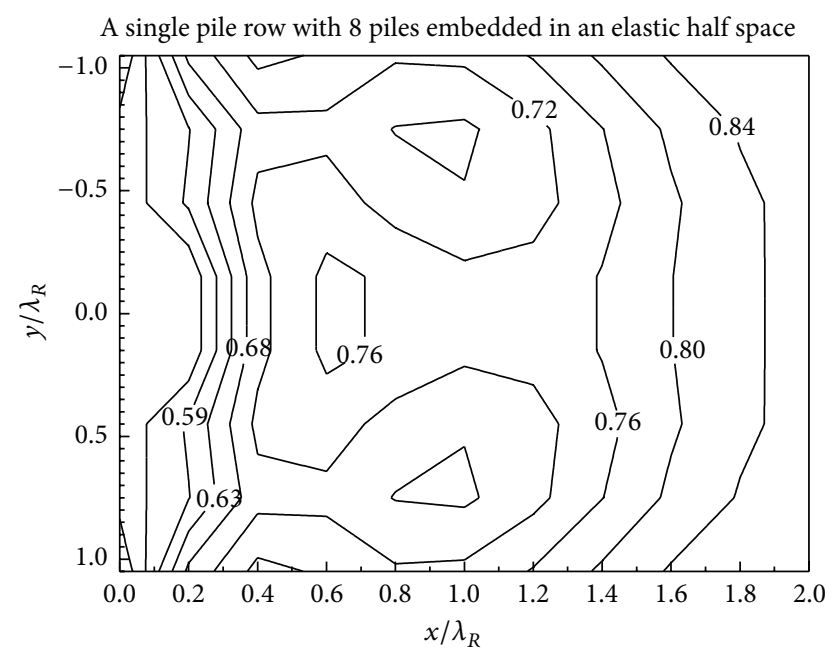

FIgURE 3: The contour of the amplitude reduction ratio $A_{r}$ for a single pile row with eight piles as vibration isolation system for the reduced elastic half space.

neighboring piles in a pile row as well as the spacing between neighboring pile rows $(q)$ on the vibration isolation effect will be discussed.

Typical values of the parameters for the viscoelastic half space are $a_{p}=a_{s}=0.7, \alpha_{p}=\alpha_{s}=0.7, \eta_{p}=\eta_{s}=0.1 \mathrm{~s}$, $M_{\text {os }}=2.0 \times 10^{7} \mathrm{~N} / \mathrm{m}^{2}, M_{\infty p}=6.0 \times 10^{7} \mathrm{~N} / \mathrm{m}^{2}$, and $\rho=2.0 \times$ $10^{3} \mathrm{~kg} / \mathrm{m}^{3}$. The reference length is $\lambda_{R}=5.0 \mathrm{~m}$. The vibration source is a moving distributed load with a constant speed $\mathrm{c}$ in the negative direction of the $y$-axis. The intensity of the load is $100 \mathrm{kN}$, and it is uniformly distributed over a rectangle $2 a \times$ $2 b=0.8 \mathrm{~m} \times 0.8 \mathrm{~m}$ with a vibration frequency $f=0$. The load moves along a line parallel to the $y$-axis at a constant speed $c=0.5 v_{s 0}$ in the negative direction of the $y$-axis, where $v_{s 0}=$ $\sqrt{M_{\text {os }} / \rho}$. The distance between the center of the first rows piles and the center of the distributed load is $d_{s}=3.0 \mathrm{~m}$. Each pile has the same diameter $d=0.8 \mathrm{~m}$ and the same density $\rho_{p}=3.9 \times 10^{3} \mathrm{~kg} / \mathrm{m}^{3}$. Each pile has the same length $L=$ $10.0 \mathrm{~m}$ and the same Young's modulus $E_{p}=100 E_{s 0}\left(E_{s 0}=\right.$ $\left.M_{\infty \mathrm{s}} \times\left(3 M_{\infty \mathrm{p}}-4 M_{\infty \mathrm{s}}\right) /\left(M_{\infty \mathrm{p}}-M_{\infty \mathrm{s}}\right)\right)$. Also, the net spacing between two neighboring piles is $s=0.25 \mathrm{~m}$ and the spacing between the two adjacent pile rows is $q=0.5 \mathrm{~m}$.

Note that when the influence of one parameter is examined, all the other parameters will take the typical values as given above.

4.2.1. Effects of the Speed of the Moving Load. In this section, the influences of the speed of the moving load $(c)$ on the vibration isolation effect of the pile rows will be examined. In the calculation, four different moving load speeds $c=0.2 v_{s 0}$, $c=0.5 v_{s 0}, c=0.9 v_{s 0}$, and $c=1.2 v_{s 0}$ are considered, where $v_{s 0}=\sqrt{M_{\infty \mathrm{os}} / \rho}$, while the remainder parameters for the vibration source and the viscoelastic half space take the typical values as given above. Also, two piles rows embedded in the viscoelastic half space are used as the passive vibration isolation system. Thus, the number of pile rows is $K=2$ and the numbers of piles in the pile rows are $n_{1}=9$ and $n_{2}=8$, respectively.

Figures 4(a)-4(d) illustrate the variation of the amplitude reduction ratio $A_{r}$ on the surface of the half space at the instant when the moving load passing the point $(x, y, z)=$ $(-3.0 \mathrm{~m}, 0 \mathrm{~m}, 0 \mathrm{~m})$ with speeds $c=0.2 v_{s 0}, c=0.5 v_{s 0}$, $c=0.9 v_{s 0}$, and $c=1.2 v_{s 0}$, respectively. From Figure 4, one can see that moving load speed has some influence on the effect of the isolation vibration. With the increasing moving load speed, the vibration isolation effectiveness is enhanced pronouncedly when the speed of the moving load is lower than the shear speed of the viscoelastic half space. It is also shown in Figure 4(d) that the amplitude reduction ratio at the instant when the moving load is located at the point $(x, y, z)=(-3.0 \mathrm{~m}, 0 \mathrm{~m}, 0 \mathrm{~m})$ becomes asymmetrical with respect to $x$ axis: for the case of $c=1.2 v_{s 0}$, the vibration isolation effect for the domain $y>0$ is better than that for $y<0$.

According to our model, the average amplitude reduction ratios $A_{r v}$ for the present Cole-Cole viscoelastic half space and the reduced elastic medium corresponding to the instant when the moving load with different velocity passing through the point $(x, y, z)=(-3.0 \mathrm{~m}, 0 \mathrm{~m}, 0 \mathrm{~m})$ is derived. For the case of the Cole-Cole viscoelastic half-space model, the average amplitude reduction ratios $A_{r v}$ are equal to 0.8491 , $0.8346,0.8024$, and 0.4941 for the moving load with velocities $c=0.2 v_{s 0}, c=0.5 v_{s 0}, c=0.9 v_{s 0}$, and $c=1.2 v_{s 0}$, respectively, while for the case of the reduced elastic medium, the corresponding $A_{r v}$ is equal to $0.852,0.8476,0.7526$, and 0.6213 for $c=0.2 v_{s 0}, c=0.5 v_{s 0}, c=0.9 v_{s 0}$, and $c=1.2 v_{s 0}$, respectively. Thus, we can see that for the same pile row and the same vibration source, the vibration isolation effect of the Cole-Cole viscoelastic half-space model is better than that for the elastic medium, particularly on the case of moving load with high velocity.

4.2.2. Effects of the Number of the Pile Rows. In this example, we consider the following three pile rows embedded in the viscoelastic half space: a single pile row with 9 piles $(K=1$, $\left.n_{1}=9\right)$; two pile rows with 9 and 8 piles $\left(K=2, n_{1}=9\right.$, $\left.n_{2}=8\right)$, respectively; three pile rows with 9,8 , and 9 piles $\left(K=3, n_{1}=9, n_{2}=8, n_{3}=9\right)$, respectively (Figure 6).

Figures 6(a) and 6(b) plot the variation of the amplitude reduction ratio $A_{r}$ on the surface of the viscoelastic half space at the instant when the moving load with the velocity $c=$ $0.5 v_{s 0}$ passing through the point $(x, y, z)=(-3.0 \mathrm{~m}, 0 \mathrm{~m}, 0 \mathrm{~m})$ for the two kinds of pile rows ( $K=1$ and $K=3$ ). Note that the results for the case of two pile rows with 9 and 8 piles ( $K=2, n_{1}=9$, and $\left.n_{2}=8\right)$ have already been presented in Section 4.2.1 (see Figure 4(b)).

As expected, it follows from Figure 6 that the increase of the number of pile rows will enhance the vibration isolation effect of the pile rows. The average amplitude reduction ratios at the instant when the moving load with the velocity $c=$ $0.5 v_{s 0}$ passing the point $(x, y, z)=(-3.0 \mathrm{~m}, 0 \mathrm{~m}, 0 \mathrm{~m})$ for the single pile row, the two pile rows and the three pile rows are $A_{r v}=0.9189,0.8346$, and 0.7769 , respectively. 


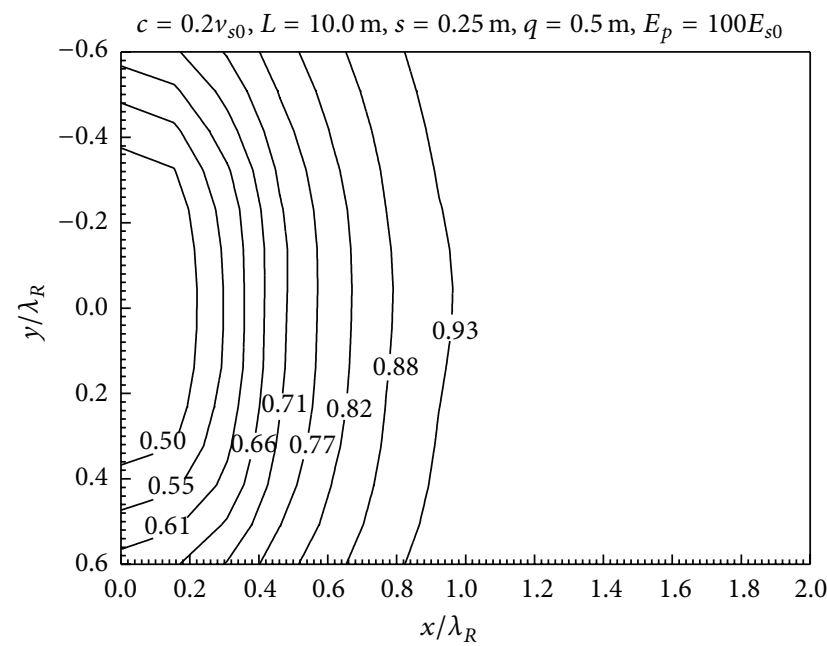

(a)

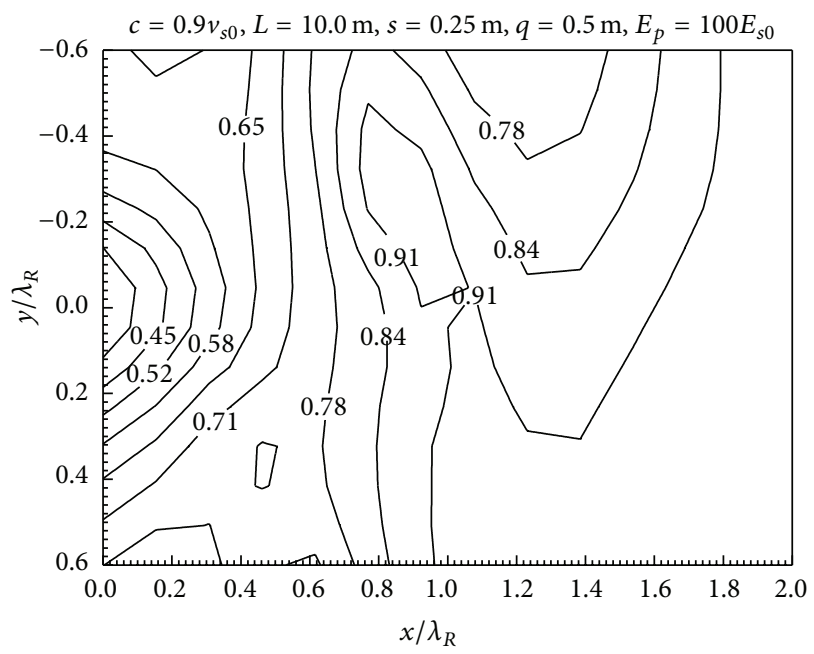

(c)

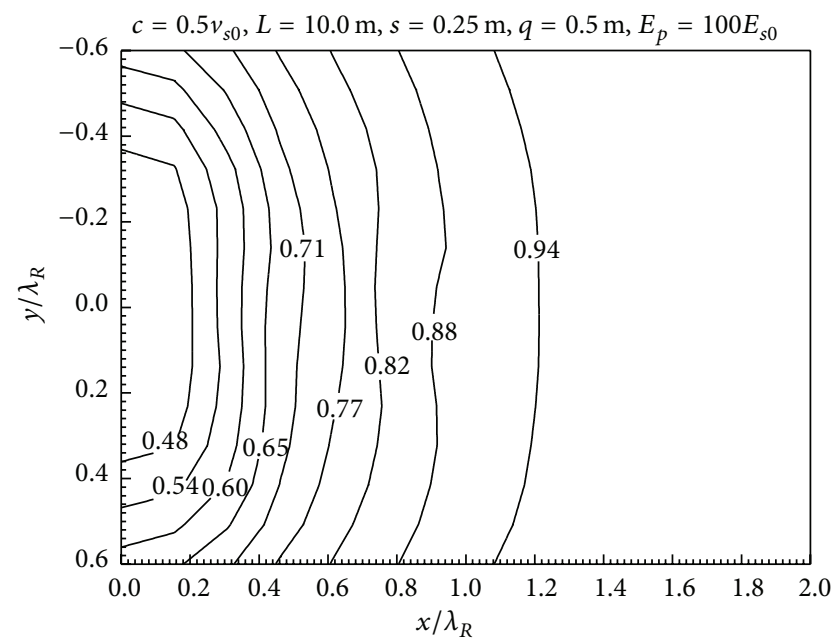

(b)

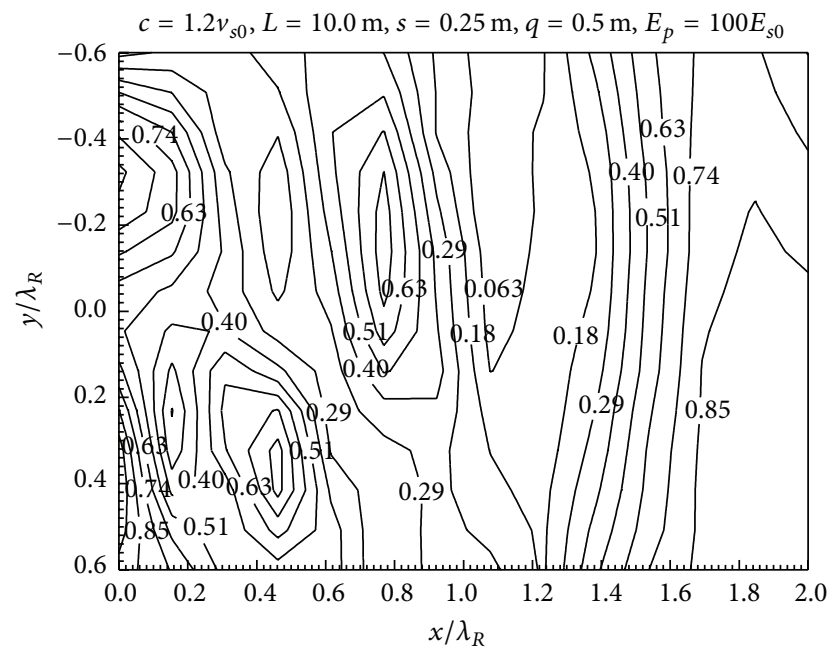

(d)

FIgURE 4: The variation of the amplitude reduction ratio $A_{r}$ on the surface for the viscoelastic half space at the instant when the moving load passing the point $(x, y, z)=(-3.0 \mathrm{~m}, 0 \mathrm{~m}, 0 \mathrm{~m})$ with four different speeds: (a) $c=0.2 v_{s 0}$; (b) $c=0.5 v_{s 0}$; (c) $c=0.9 v_{s 0}$; (d) $c=1.2 v_{s 0}$, where $v_{s 0}=\sqrt{M_{\infty} / \rho}$.

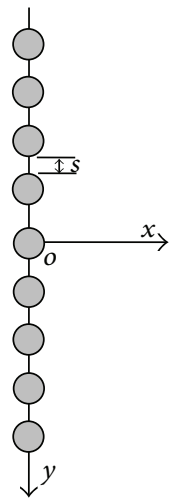

(a)

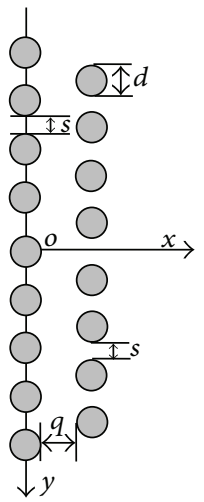

(b)

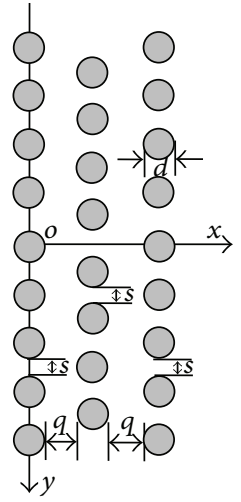

(c)

FIGURE 5: Multiple pile rows embedded in a viscoelastic half space as vibration isolation system against the vibration due to a harmonic moving load: (a) a single pile row; (b) two pile rows; (c) three pile rows. 


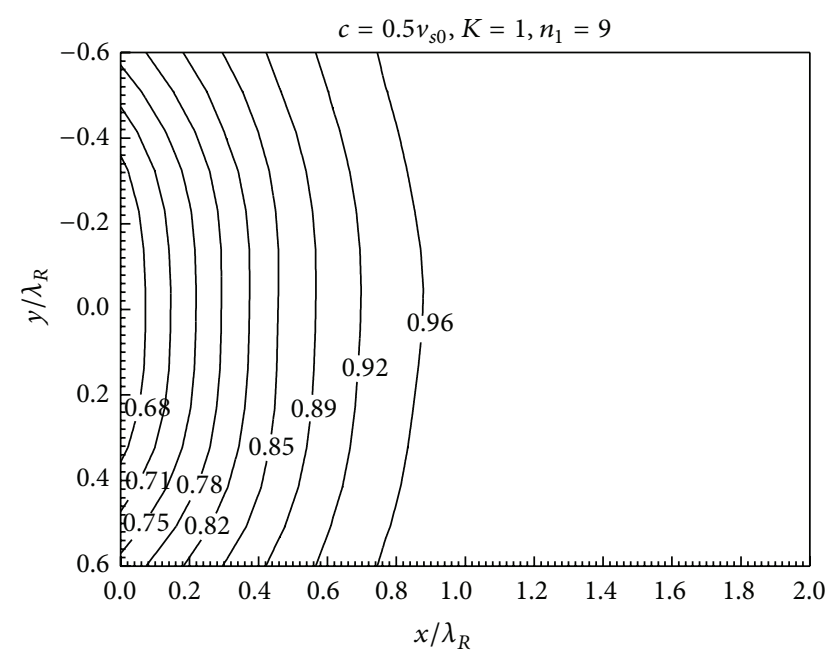

(a)

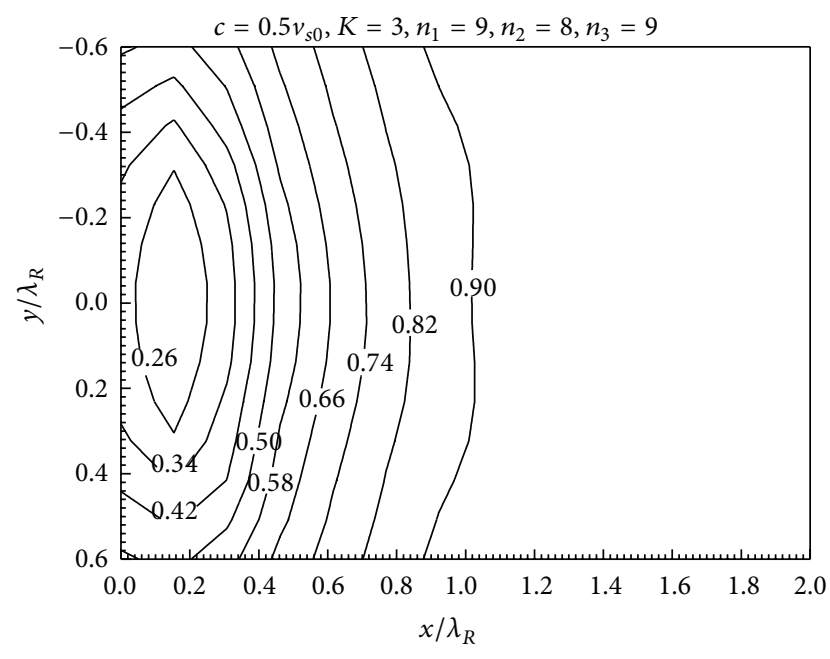

(b)

Figure 6: The variation of the amplitude reduction ratio $A_{r}$ on the surface of the viscoelastic half space at the instant when the moving load passing the point $(x, y, z)=(-3.0 \mathrm{~m}, 0 \mathrm{~m}, 0 \mathrm{~m})$ with the speed $c=0.5 v_{s 0}$ for the two kinds of pile rows: (a) a single pile row $\left(K=1, n_{1}=9\right)$; (b) three piles rows $\left(K=3, n_{1}=9, n_{2}=8, n_{3}=9\right)$.

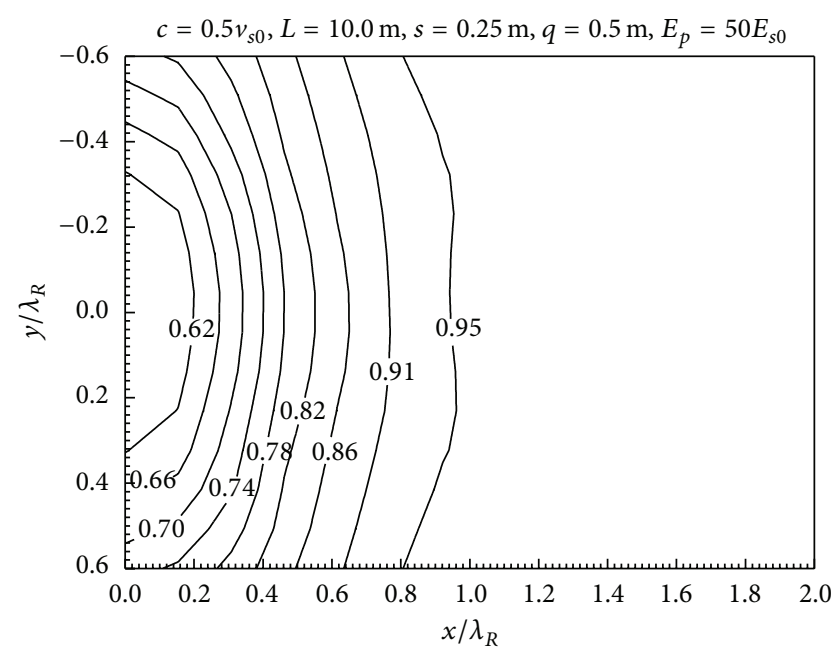

(a)

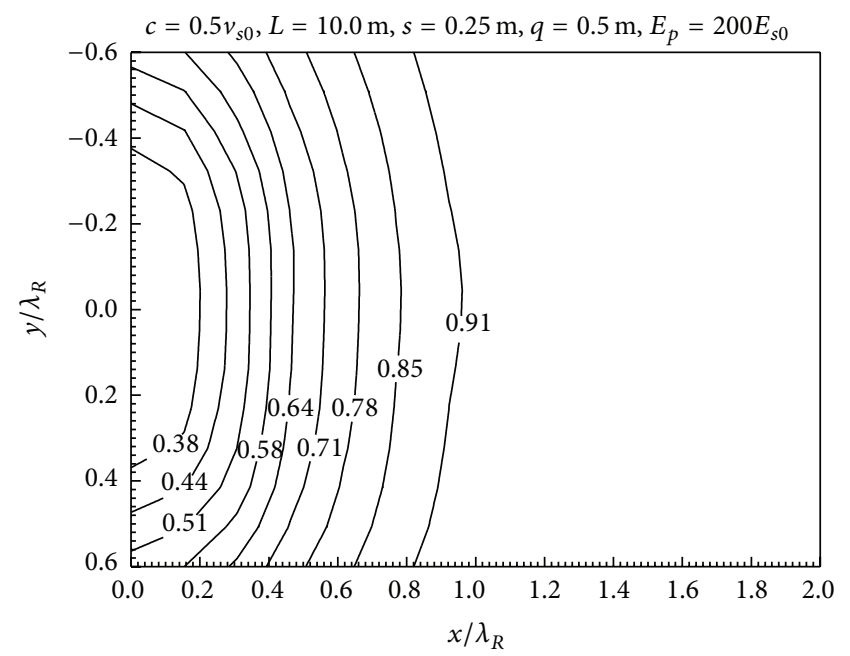

(b)

FIGURE 7: The variation of the amplitude reduction ratio $A_{r}$ on the surface of the viscoelastic half space at the instant when the moving load passing the point $(x, y, z)=(-3.0 \mathrm{~m}, 0 \mathrm{~m}, 0 \mathrm{~m})$ with speed $c=0.5 v_{s 0}$ for two cases of $E_{p} / E_{s 0}$ : (a) $E_{p} / E_{s 0}=50$; (b) $E_{p} / E_{s 0}=200$, where $E_{s 0}=M_{\infty \mathrm{s}} \times\left(3 M_{\infty \mathrm{p}}-4 M_{\infty \mathrm{s}}\right) /\left(M_{\infty \mathrm{p}}-M_{\infty \mathrm{s}}\right)$.

4.2.3. Effects of Young's Modulus $\left(E_{p}\right)$ of the Piles. Young's modulus $\left(E_{p}\right)$ of the pile is an important parameter for the design of the pile row vibration isolation system. Herein, the influence of Young's modulus of pile on the vibration isolation effect will be investigated. As previously mentioned, in this example, two pile rows ( $\left.K=2, n_{1}=9, n_{2}=8\right)$ embedded in the viscoelastic half space are used to screen the vibration due to the moving load. Young's modulus of the piles takes three different values to make $E_{p} / E_{s 0}=50,100$, and 200, respectively, with $E_{s 0}=M_{\infty s} \times\left(3 M_{\infty p}-4 M_{\infty s}\right) /\left(M_{\infty p}-\right.$ $\left.M_{\mathrm{os}}\right)$.
Figures $7(\mathrm{a})$ and $7(\mathrm{~b})$ show the variation of the amplitude reduction ratio $A_{r}$ on the surface of the viscoelastic half space for $E_{p} / E_{s 0}=50$ and 200 when the moving load with the velocity $c=0.5 v_{s 0}$ passing the point $(x, y, z)=(-3.0 \mathrm{~m}, 0 \mathrm{~m}, 0 \mathrm{~m})$. Note that the result for $E_{p} / E_{s 0}=100$ is presented in Section 4.2.1 (see Figure 4(b)). The average amplitude reduction ratio $A_{r v}$ at the instant when the moving load with the velocity $c=0.5 v_{s 0}$ passing the point $(x, y, z)=$ $(-3.0 \mathrm{~m}, 0 \mathrm{~m}, 0 \mathrm{~m})$ for the three modulus ratios is. for $E_{p} / E_{s 0}=50, A_{r v}=0.8896$; for $E_{p} / E_{s 0}=100, A_{r v}=$ 0.8346 ; for $E_{p} / E_{s 0}=200, A_{r v}=0.8146$. One can see clearly 


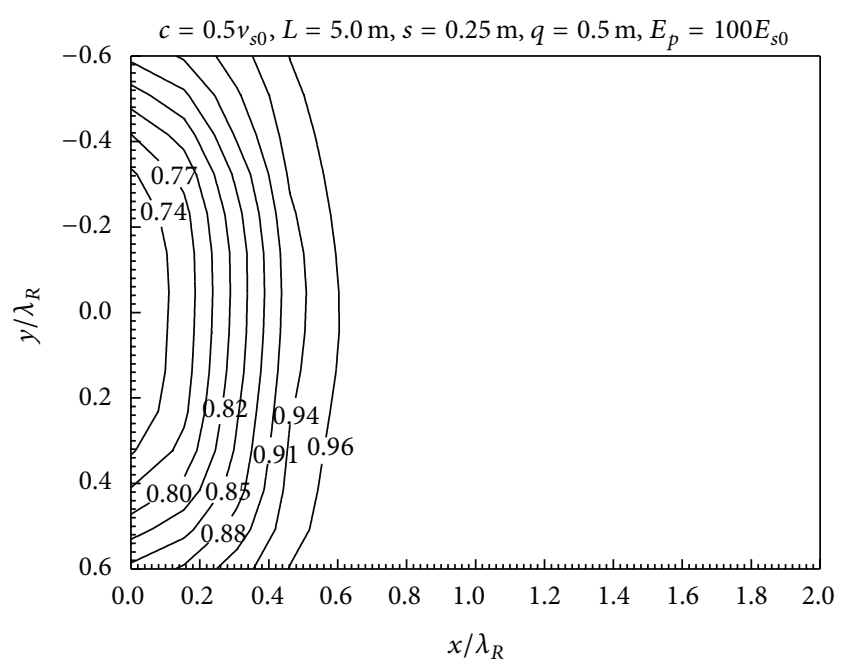

(a)

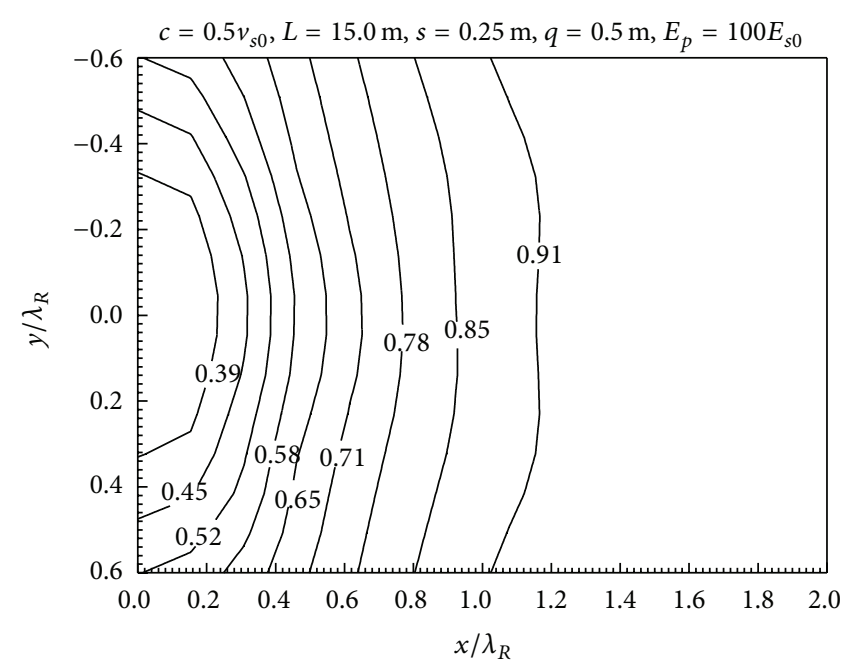

(b)

FIGURE 8: The variation of the amplitude reduction ratio $A_{r}$ on the surface of the viscoelastic half space at the instant when the moving load with speed $c=0.5 v_{s 0}$ passing the point $(x, y, z)=(-3.0 \mathrm{~m}, 0 \mathrm{~m}, 0 \mathrm{~m})$ for the two cases of pile length (a) $L=5.0 \mathrm{~m} ;$ (b) $L=15.0 \mathrm{~m}$.

that the average amplitude reduction ratio $A_{r v}$ decreases with increasing $E_{p} / E_{s 0}$, which suggest stiffer pile rows will produce a better vibration isolation effect.

4.2.4. Effects of the Length of the Piles. The influence of the length of the piles on the vibration isolation effect of pile rows is examined in this section. As in Section 4.2.1, two pile rows ( $\left.K=2, n_{1}=9, n_{2}=8\right)$ embedded in the viscoelastic half space are used to isolate the vibration due to the moving load. To check the influence of the pile length, the pile length takes the following values: $L=5.0 \mathrm{~m}, 10.0 \mathrm{~m}$, and $15.0 \mathrm{~m}$, respectively.

Figures 8(a) and 8(b) show the variation of the amplitude reduction ratio $A_{r}$ on the surface of the viscoelastic half space for $L=5.0 \mathrm{~m}$ and $15.0 \mathrm{~m}$ when the moving load with the velocity $c=0.5 v_{s 0}$ passing the point $(x, y, z)=$ $(-3.0 \mathrm{~m}, 0 \mathrm{~m}, 0 \mathrm{~m})$. Note that the result for $L=10.0 \mathrm{~m}$ has been presented in Section 4.2.1 (see Figure 4(b)). The average amplitude reduction ratio $A_{r v}$ at the instant when the moving load with the velocity $c=0.5 v_{s 0}$ passing the point $(x, y, z)=$ $(-3.0 \mathrm{~m}, 0 \mathrm{~m}, 0 \mathrm{~m})$ for $L=5.0 \mathrm{~m}, 10.0 \mathrm{~m}$ and $15.0 \mathrm{~m}$ is equal to $0.9463,0.8346$, and 0.7927 , respectively. It follows that the length of piles has a significant influence on the average amplitude reduction ratios $A_{r v}$ : pile rows with a larger pile length usually produce a better vibration isolation effect than shorter ones.

4.2.5. Effects of the Pile Net Spacing. The net spacing (s) between neighboring piles in a pile row is an important parameter for the design of pile rows. In this section, two rows piles $\left(K=2, n_{1}=9, n_{2}=8\right)$ embedded in the viscoelastic half space are used to investigate the influence of the net spacing. To examine the influence of the net spacing $s$ between neighboring piles, the net spacing $s$ takes the values $0.25 \mathrm{~m}, 0.5 \mathrm{~m}$, and $1.0 \mathrm{~m}$, respectively.
Figures $9(a)$ and $9(b)$ show the variation of the amplitude reduction ratio $A_{r}$ on the surface of the viscoelastic half space for $s=0.5 \mathrm{~m}$ and $1.0 \mathrm{~m}$ when the moving load with the velocity $c=0.5 v_{s 0}$ is passing the point $(x, y, z)=$ $(-3.0 \mathrm{~m}, 0 \mathrm{~m}, 0 \mathrm{~m})$. The result for the case of $s=0.25 \mathrm{~m}$ has already been given in Section 4.2.1 (see Figure 4(b)). It clearly indicates the decay of the vibration isolation effect behind row pile with increasing net spacing $s$. The average amplitude reduction ratio $A_{r v}$ at the instant when the moving load with the velocity $c=0.5 v_{s 0}$ passing the point $(x, y, z)=$ $(-3.0 \mathrm{~m}, 0 \mathrm{~m}, 0 \mathrm{~m})$ for $s=0.25 \mathrm{~m}, 0.5 \mathrm{~m}$ and $1.0 \mathrm{~m}$ is $A_{r v}$ $=0.8346,0.8804$, and 0.9103 , respectively, which shows that the average amplitude reduction ratio $A_{r v}$ has considerable increase with increasing net spacing $s$. Thus, it can be concluded that a smaller net pile spacing usually leads to a better vibration isolation effect.

4.2.6. Effects of the Spacing between Adjacent Pile Rows. To examine the effect of the spacing $(q)$ between neighboring pile rows, as in Section 4.2.1, two pile rows $\left(K=2, n_{1}=\right.$ $9, n_{2}=8$ ) embedded in the viscoelastic half space are used as an example. To examine the influence of the spacing between neighboring pile rows, $q$ takes $0.5 \mathrm{~m}, 1.0 \mathrm{~m}$, and $1.5 \mathrm{~m}$, respectively.

Figures 10(a) and 10(b) show the variation of the amplitude reduction ratio $A_{r}$ on the surface of the viscoelastic half space for $q=1.0 \mathrm{~m}$ and $1.5 \mathrm{~m}$ when the moving load with the velocity $c=0.5 v_{s 0}$ is passing the point $(x, y, z)=$ $(-3.0 \mathrm{~m}, 0 \mathrm{~m}, 0 \mathrm{~m})$. The result for the case of $q=0.5 \mathrm{~m}$ has already been given in Section 4.2.1 (see Figure 4(b)). The average amplitude reduction ratio $A_{r v}$ at the instant when the moving load located at the point $(x, y, z)=$ $(-3.0 \mathrm{~m}, 0 \mathrm{~m}, 0 \mathrm{~m})$ for $q=0.5 \mathrm{~m}, 1.0 \mathrm{~m}$ and $1.5 \mathrm{~m}$ is equal to $A_{r v}=0.8346,0.8352$, and 0.8356 , respectively, which indicates that, compared with other parameters, the space between 


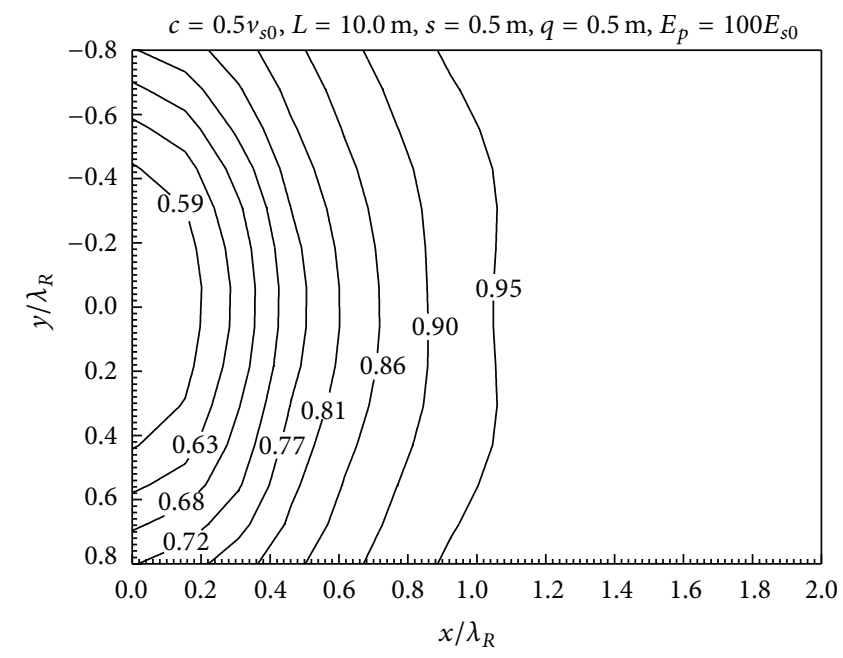

(a)

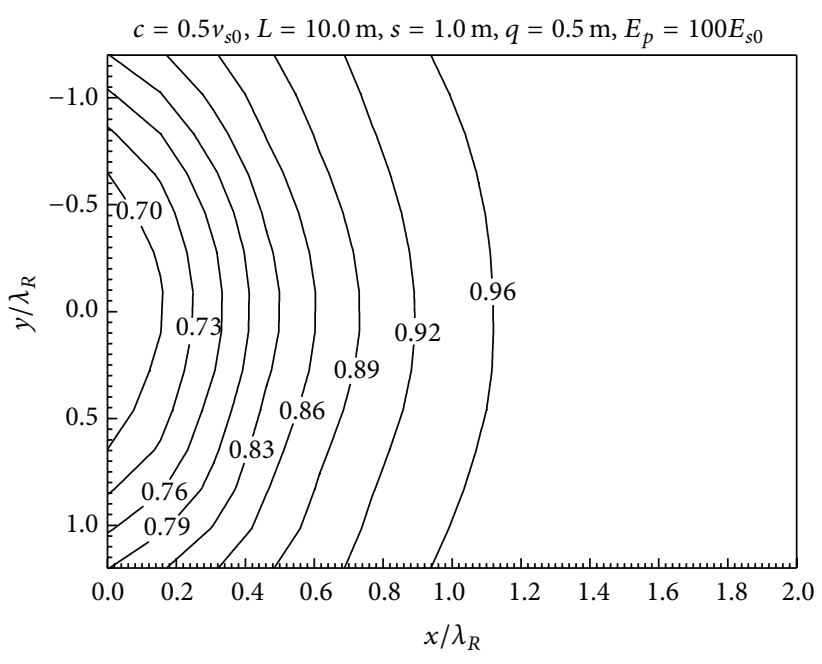

(b)

FIGURE 9: The variation of the amplitude reduction ratio $A_{r}$ on the surface of the viscoelastic half space at the instant when the moving load passing the point $(x, y, z)=(-3.0 \mathrm{~m}, 0 \mathrm{~m}, 0 \mathrm{~m})$ with speed $c=0.5 v_{s 0}$ for the two cases of the net spacing: (a) $s=0.5 \mathrm{~m} ;(\mathrm{b}) s=1.0 \mathrm{~m}$.

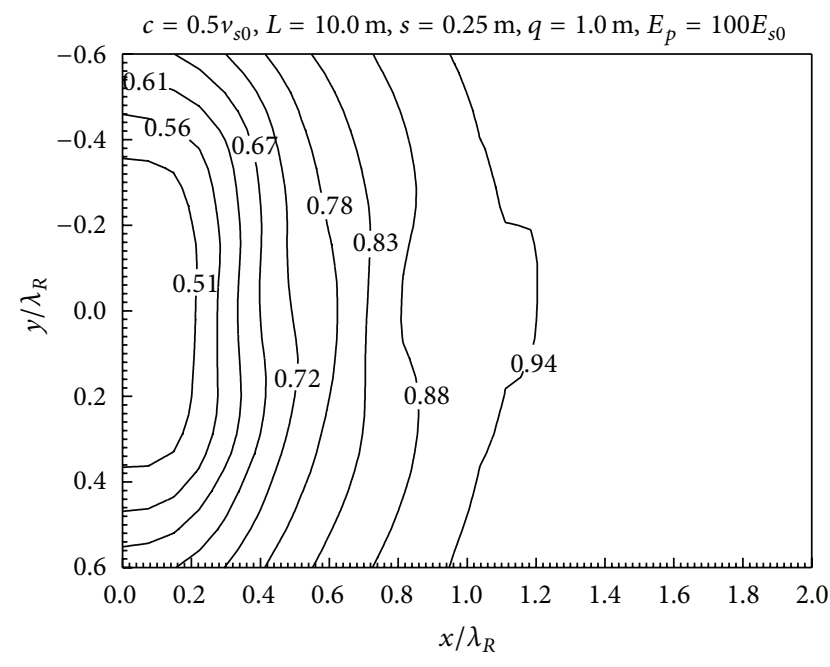

(a)

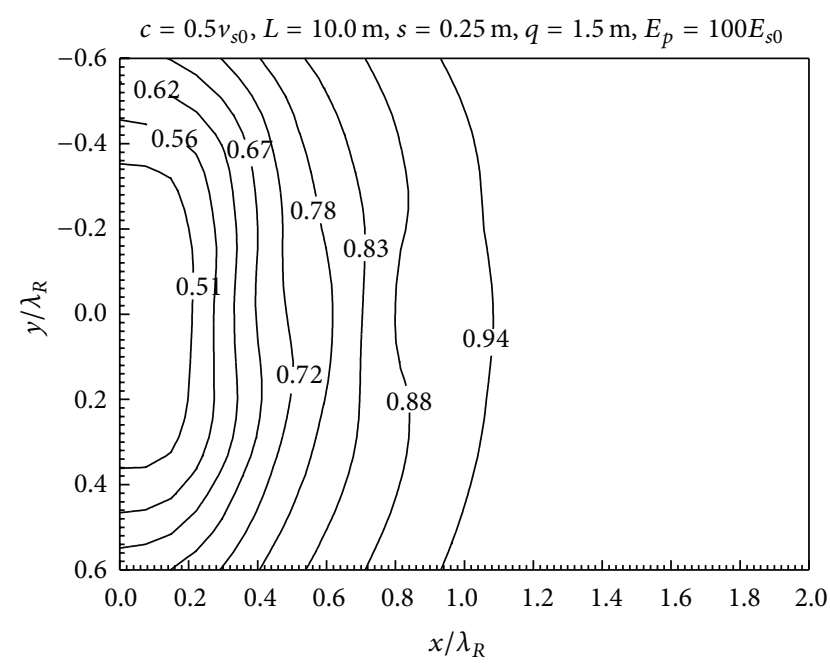

(b)

FIGURE 10: The variation of the amplitude reduction ratio $A_{r}$ on the surface of the viscoelastic half space at the instant when the moving load passing the point $(x, y, z)=(-3.0 \mathrm{~m}, 0 \mathrm{~m}, 0 \mathrm{~m})$ with speed $c=0.5 v_{s 0}$ for the two cases of the net spacing: (a) $q=1.0 \mathrm{~m}$; (b) $q=1.5 \mathrm{~m}$.

two adjacent pile rows has a relatively smaller effect on the vibration isolation effect of pile rows.

\section{Conclusions}

The numerical simulation of the isolation of vibration due to a harmonic moving load applied on the surface of a viscoelastic half space by pile rows has been carried out in this study (Figure 5). The viscoelastic half space is characterized by the Cole-Cole model. The semianalytical nature of the proposed method circumvents the discretization of the whole calculation domain, thus reducing the CPU time for the current problem substantially. Consequently, the proposed method is important for the design of vibration isolation and can be used to assess the efficiency of pile rows as a barrier system against ground vibrations. To verify the proposed method, result of this study was compared with existing results, which shows the proposed solution is in a good agreement with the existing result.

To study the vibration isolation effect of pile rows, the influences of the moving load speed, Young's modulus of the pile, the pile length, and the spacing between piles as well as the spacing between neighboring pile rows have been investigated. Based on the numerical simulations performed in this study, the following conclusions can be drawn.

(i) The same pile rows can achieve a better vibration isolation effect for higher speed loads than for lower speed loads. 
(ii) Young's modulus of the pile is an important parameter affecting the vertical vibration isolation effects of pile rows. Increasing Young's modulus of the pile rows will enhance the vibration isolation effect.

(iii) Pile length is the most important factor for the vertical vibration isolation effect of pile rows. Generally, pile rows with a longer pile length have a better vibration isolation effect than those with a shorter pile length.

(iv) The net spacing between neighboring piles is crucial for the vibration isolation effect of pile rows. Generally, to obtain a better vibration isolation effect for high speed loads, the net spacing should take a smaller value.

(v) A good vibration isolation effect can be realized by increasing the number of pile rows and reducing the net spacing between neighboring piles.

\section{Conflict of Interests}

The authors declare that there is no conflict of interests regarding the publication of this paper.

\section{Acknowledgments}

The project is supported by the National Natural Science Foundation of China with Grant no. 51269021 and Key Project of Natural Science Foundation of Jiangxi Province with Grant no. 20133ACB20006.

\section{References}

[1] K. Emad and G. D. Manolis, "Shallow trenches and propagation of surface waves," Journal of Engineering Mechanics, vol. 111, no. 2, pp. 279-282, 1985.

[2] Z. Cao, Y. Cai, A. Boström, and J. Zheng, "Semi-analytical analysis of the isolation to moving-load induced ground vibrations by trenches on a poroelastic half-space," Journal of Sound and Vibration, vol. 331, no. 4, pp. 947-961, 2012.

[3] Y. Cai, G. Ding, C. Xu, and J. Wang, "Vertical amplitude reduction of Rayleigh waves by a row of piles in a poroelastic half-space," International Journal for Numerical and Analytical Methods in Geomechanics, vol. 33, no. 16, pp. 1799-1821, 2009.

[4] S. E. Kattis, D. Polyzos, and D. E. Beskos, "Modelling of pile wave barriers by effective trenches and their screening effectiveness," Soil Dynamics and Earthquake Engineering, vol. 18, no. 1, pp. 110, 1999.

[5] S. E. Kattis, D. Polyzos, and D. E. Beskos, "Vibration isolation by a row of piles using a 3-D frequency domain BEM," International Journal for Numerical Methods in Engineering, vol. 46, no. 5, pp. 713-728, 1999.

[6] P. Tsai, Z. Feng, and T. Jen, “Three-dimensional analysis of the screening effectiveness of hollow pile barriers for foundationinduced vertical vibration," Computers and Geotechnics, vol. 35, no. 3, pp. 489-499, 2008.

[7] R. Muki and E. Sternberg, "Elastostatic load-transfer to a half-space from a partially embedded axially loaded rod," International Journal of Solids and Structures, vol. 6, no. 1, pp. 69-90, 1970.
[8] J. Lu, B. Xu, and J. Wang, "A numerical model for the isolation of moving-load induced vibrations by pile rows embedded in layered porous media," International Journal of Solids and Structures, vol. 46, no. 21, pp. 3771-3781, 2009.

[9] J. Lu, D. Jeng, J. Wan, and J. Zhang, "A new model for the vibration isolation via pile rows consisting of infinite number of piles," International Journal for Numerical and Analytical Methods in Geomechanics, vol. 37, pp. 2394-2426, 2013.

[10] Y. N. Rabotnov, Elements of Hereditary Solid Mechanics, Mir Publication, Moscow, Russia, 1980.

[11] B. Nolte, S. Kempfle, and I. Schäfer, "Does a real material behave fractionally? Applications of fractional differential operators to the damped structure borne sound in viscoelastic solids," Journal of Computational Acoustics, vol. 11, no. 3, pp. 451-489, 2003.

[12] K. S. Cole and R. H. Cole, "Dispersion and absorption in dielectrics I. Alternating current characteristics," The Journal of Chemical Physics, vol. 9, no. 4, pp. 341-351, 1941.

[13] R. L. Bagley and P. J. Torvik, "On the fractional calculus model of viscoelastic behavior," Journal of Rheology, vol. 30, no. 1, pp. 133-155, 1986.

[14] J. Lu and A. Hanyga, "Numerical modelling method for wave propagation in a linear viscoelastic medium with singular memory," Geophysical Journal International, vol. 159, no. 2, pp. 688-702, 2004.

[15] M. Soula, T. Vinh, and Y. Chevalier, "Transient responses of polymers and elastomers deduced from harmonic responses," Journal of Sound and Vibration, vol. 205, no. 2, pp. 185-203, 1997.

[16] T. D. Jones, "Pore fluids and frequency-dependent wave propagation in rocks," Geophysics, vol. 51, no. 10, pp. 1939-1953, 1986.

[17] M. Batzle, R. Hofmann, D. Han, and J. Castagna, "Fluids and frequency dependent seismic velocity of rocks," Leading Edge, vol. 20, no. 2, pp. 168-171, 2001.

[18] G. F. Fowler and G. B. Sinclair, "The longitudinal harmonic excitation of a circular bar embedded in an elastic half-space," International Journal of Solids and Structures, vol. 14, no. 12, pp. 999-1012, 1978.

[19] R. Y. S. Pak and P. C. Jennings, "Elasticdynamic response of pile under transverse excitations," Journal of Engineering Mechanics, vol. 113, no. 7, pp. 1101-1116, 1987.

[20] A. V. Oppenheim and R. W. Schafer, Discrete-Time Signal Processing, Prentice-Hall, Englewood Cliffs, NJ, USA, 1999.

[21] R. D. Woods, "Screening of surface waves in soils," Journal of Soil Mechanics and Foundation Engineering, vol. 94, no. 4, pp. 951-979, 1968. 


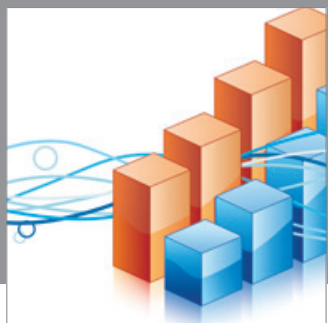

Advances in

Operations Research

mansans

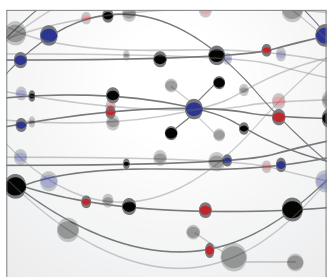

The Scientific World Journal
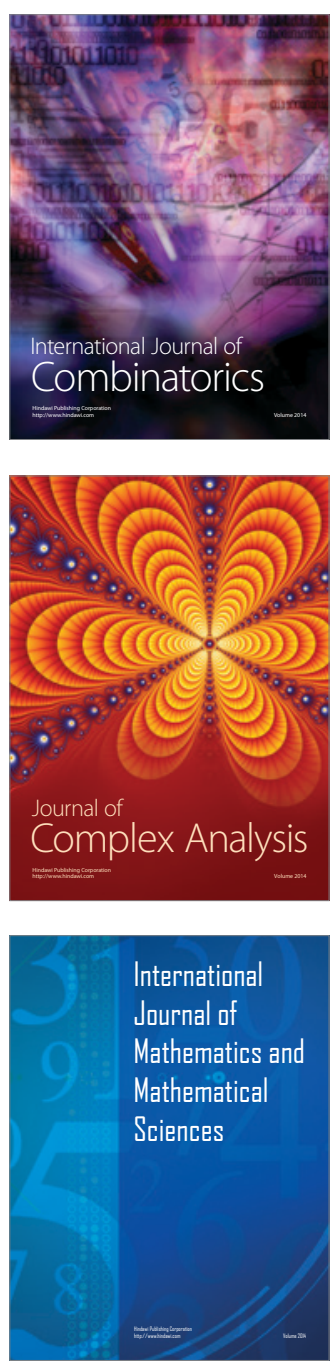
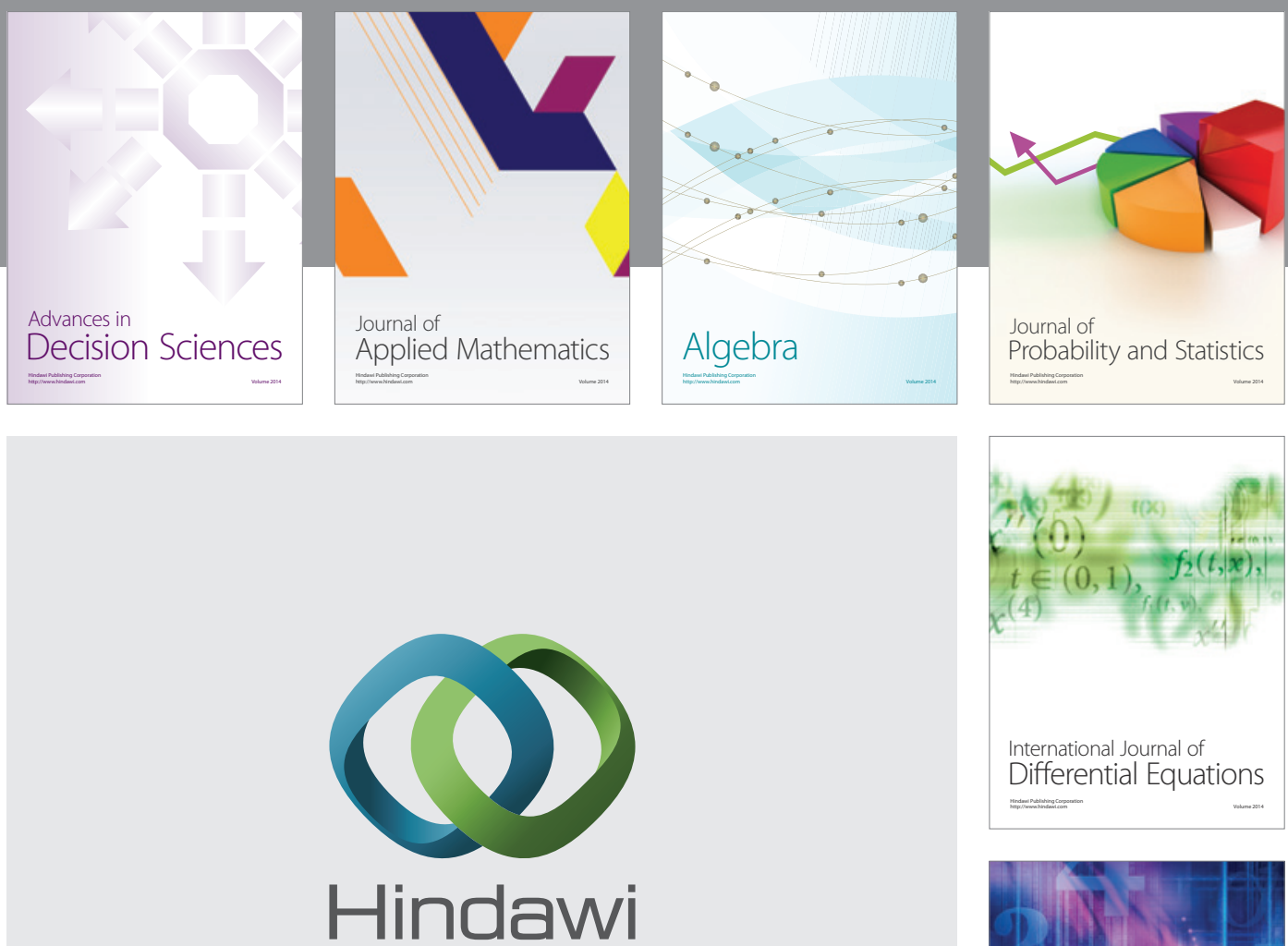

Submit your manuscripts at http://www.hindawi.com
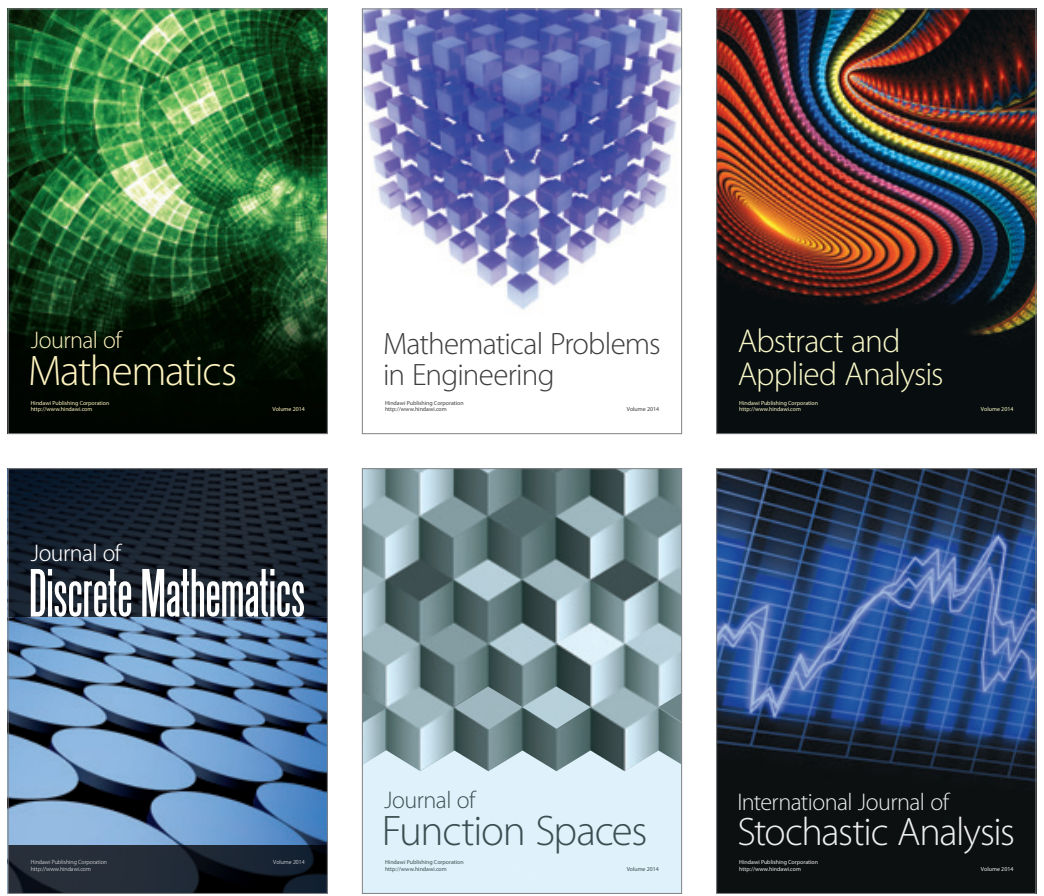

Journal of

Function Spaces

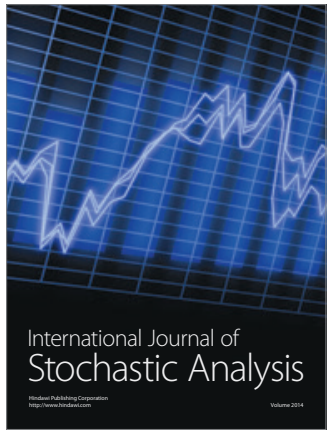

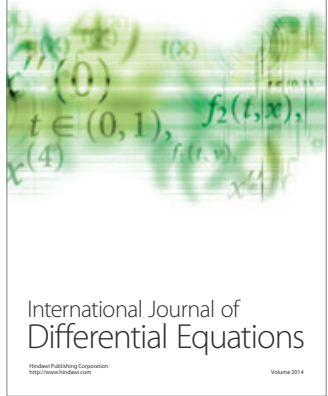
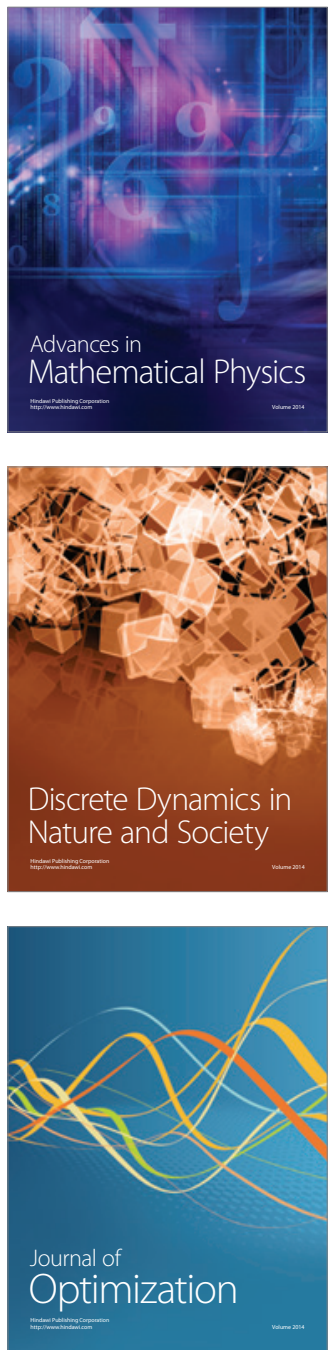